\title{
Riemann and Klein Surfaces with Nodes Viewed as Quotients
}

\author{
Ignacio C. GARIJO \\ Dpto. de Matemáticas Fundamentales \\ Facultad de Ciencias, UNED \\ c/ Senda del Rey, 9 \\ 28040 Madrid - Spain \\ igarijo@mat.uned.es
}

Received: March 10, 2005

Accepted: October 10, 2005

\begin{abstract}
If $G$ is a group of automorphisms that acts properly discontinuously on a Riemann or Klein surface $X$, then there exists a unique structure of Riemann or Klein surface on $X / G$ such that the projection $\pi: X \rightarrow X / G$ is a morphism. The analogous result is not true when we deal with surfaces with nodes. In this paper we give a new definition of a group that acts properly discontinuously on a surface with nodes in order to obtain a similar theorem.
\end{abstract}

Key words: Riemann surfaces with nodes, Klein surfaces with nodes, groups of automorphisms.

2000 Mathematics Subject Classification: 30F50, 30F10, 20H10, 20H15.

\section{Introduction}

Riemann surfaces with nodes appear as compactification points of the Deligne-Mumford compactification of the moduli space of compact and connected smooth complex curves of given genus [2]. These compactifications are a useful tool in recent important progress in mathematics. Concretely, the proof of Witten's conjecture given by M. Kontsevich (see $[6,7,10]$ ) uses, in an essential way, the cell decomposition of the spaces $\overline{\mathcal{M}}_{g, n} \times \mathbb{R}_{+}^{n}$, where $\overline{\mathcal{M}}_{g, n}$ is the Deligne-Mumford compactification by Riemann surfaces with nodes of the moduli space of compact and connected smooth complex curves of genus $g$ with $n$ marked points. 
The moduli space of compact and connected smooth real algebraic curves of given genus $g$, denoted $\mathcal{M}_{\mathbb{R}}^{g}$, is neither compact nor connected. In [9], M. Seppälä constructs moduli spaces of compact and connected stable symmetric Riemann surfaces and proves that these spaces are compact and connected. In other words, he constructs a compactification of $\mathcal{M}_{\mathbb{R}}^{g}$ using symmetric Riemann surfaces with nodes. In [3] we construct moduli spaces of compact and connected stable Klein surfaces. This way we obtain compactifications of $\mathcal{M}_{\mathbb{R}}^{g}$ with less points than those given by M. Seppälä adding symmetric Riemann surfaces with nodes. Moreover, these spaces are connected too. As an example, let us look at two families of compact and connected smooth complex algebraic curves:

$$
\begin{aligned}
A_{t} \equiv \text { The hyperelliptic curve defined by the equation } \\
\\
\quad y^{2}=\left(x^{2}-t^{2}\right)\left(x^{2}-1\right)\left(x^{2}-4\right), \\
B_{t} \equiv \text { The hyperelliptic curve defined by the equation } \\
y^{2}=\left(x^{2}+t^{2}\right)\left(x^{2}-1\right)\left(x^{2}-4\right),
\end{aligned}
$$

where $t \in(0,1)$, see [8, pag. 60-61]. If $t$ tends to 0 then

$$
\lim _{t \rightarrow 0} A_{t}=\lim _{t \rightarrow 0} B_{t}=S
$$

where $S$ is the hyperelliptic curve defined by the equation $y^{2}=x^{2}\left(x^{2}-1\right)\left(x^{2}-4\right)$. But $S$ is not a smooth curve because $(0,0)$ belongs to $S$ but there is not a neighborhood of $(0,0)$ homeomorphic to an open set of the complex plane. Indeed, this point has an neighborhood homeomorphic to

$$
\mathcal{M}=\left\{(z, w) \in \mathbb{C}^{2}|z \cdot w=0, \quad| z||<1, \quad|w|<1\right\},
$$

an is called a conic node of the surface. Furthermore, if we take the antianalytic involution $\sigma(x, y)=(\bar{x}, \bar{y})$, then $\left(A_{t}, \sigma\right)$ is a symmetric Riemann surface of topological type $(2,3,0)$ and $\left(B_{t}, \sigma\right)$ is a symmetric Riemann surface of topological type $(2,2,1)$. The limit curve $S$ is a stable symmetric Riemann surface, i.e., a surface that appears in the compactification of $\mathcal{M}_{\mathbb{R}}^{g}$. This example shows how we can use these curves for connecting the connected components of $\mathcal{M}_{\mathbb{R}}^{g}$.

On the other hand, it is a known fact that if $G$ is a group of automorphisms that acts properly discontinuously on a Riemann or Klein surface $X$, then there exists a unique structure of Riemann or Klein surface on the quotient $X / G$ such that the projection $\pi: X \rightarrow X / G$ is a morphism. In this paper, which is part of my Ph. D. Thesis [4] written under the supervision of Dr. Antonio F. Costa, we shall show that we need to adapt the definition of a group of automorphisms acting properly discontinuously on a Riemann or Klein surface with nodes in order to obtain an equivalent result.

The main problem is the existence of automorphisms $g$ that are dual on some conic node $z$, i.e., for all punctured neighborhood $U$ of $z$, the automorphism $g$ fixes each 
one of its connected components and acts analytically on one connected component of $U$ and antianalytically on the other. If our group $G$ does not contain this type of automorphism, then the quotient $X / G$ carries a structure of Klein surface with nodes such that the projection $\pi: X \rightarrow X / G$ is a morphism.

We finish the paper with Riemann-Hurwitz's Formula in the case of Riemann surfaces with nodes. This formula is an useful tool in further calculations concerning automorphisms.

\section{Riemann and Klein surfaces with nodes}

We shall define the main concepts and hence we fix the notation. All the definitions and properties we deal with in this section are exhaustively defined in [5].

A surface with nodes is a pair $S=(\Sigma, \mathcal{D})$ where $\Sigma$ is a topological Haussdorff space, $\mathcal{D} \subset \Sigma$ is a discrete set of distinguished points of $\Sigma$, and each point $z$ of $\Sigma$ has a chart $\left(U_{i}, \varphi_{i}\right)$ where $U_{i}$ is a neighborhood of $z$ and $\varphi_{i}$ is an homeomorphism between $U_{i}$ and one of the following sets:

(i) An open subset of $\mathbb{C}$,

(ii) An open subset of $\mathbb{C}^{+}=\{z \in \mathbb{C} \mid \operatorname{Im}(z) \geq 0\}$,

(iii) $\mathcal{M}=\left\{(z, w) \in \mathbb{C}^{2}|z \cdot w=0, \quad| z|<1, \quad| w \mid<1\right\}$,

(iv) $\mathcal{M}^{+}=\left\{(z, w) \in\left(\mathbb{C}^{+}\right)^{2}|z \cdot w=0, \quad| z|<1, \quad| w \mid<1\right\}$.

Moreover, if $z \in \mathcal{D}$, then $U_{i}$ is homeomorphic to an open set of $\mathbb{C}$.

If $\left\{\left(U_{i}, \varphi_{i}\right)\right\}_{i \in I}$ is an atlas of $\Sigma$, i.e., a collection of charts such that $\bigcup_{i \in I} U_{i}=\Sigma$, then we define the boundary of $\Sigma$ as

$$
\begin{aligned}
& \partial \Sigma=\left\{z \in \Sigma \mid \text { there is } i \in I \text { with } z \in U_{i}, \varphi_{i}\left(U_{i}\right) \subset \mathbb{C}^{+} \text {and } \varphi_{i}(z) \in \mathbb{R}=\partial \mathbb{C}^{+}\right\} \\
& \cup\left\{z \in \Sigma \mid \text { there is } i \in I \text { with } z \in U_{i}, \varphi_{i}\left(U_{i}\right) \subset\left(\mathbb{C}^{+}\right)^{2} \text { and } \varphi_{i}(z) \in\left(\partial\left(\mathbb{C}^{+}\right)\right)^{2}\right\} .
\end{aligned}
$$

If $z \in \Sigma$ and there is $i \in I$ with $z \in U_{i}, \varphi_{i}\left(U_{i}\right)=\mathcal{M}$ and $\varphi_{i}(z)=(0,0)$ then we say that $z$ is a conic node. We denote the set of conic nodes by $N(\Sigma, 1)$.

If $z \in \mathcal{D}$ then we say that $z$ is an inessential node. We denote the set of inessential nodes by $N(\Sigma, 2)$.

If $z \in \Sigma$ and there is $i \in I$ with $z \in U_{i}, \varphi_{i}\left(U_{i}\right)=\mathcal{M}^{+}$and $\varphi_{i}(z)=(0,0)$ then we say that $z$ is a boundary node. We denote the set of boundary nodes by $N(\Sigma, 3)$.

If $z$ belongs to $N(\Sigma)=N(\Sigma, 1) \cup N(\Sigma, 2) \cup N(\Sigma, 3)$ then we say that $z$ is a node.

Finally, we call part of $\Sigma$ each connected component of $\Sigma \backslash N(\Sigma)$.

We say that two charts, $\left(U_{i}, \varphi_{i}\right),\left(U_{j}, \varphi_{j}\right)$, have analytic (resp. dianalytic) transition if $U_{i} \cap U_{j}=\emptyset$ or the transition map

$$
\varphi_{i j}=\varphi_{j} \circ \varphi_{i}^{-1}: \varphi_{i}\left(U_{i} \cap U_{j}\right) \rightarrow \varphi_{j}\left(U_{i} \cap U_{j}\right)
$$


is analytic (resp. dianalytic) in the image under $\varphi_{i}$ of the complementary of the nodes. A Riemann surface with nodes is a pair $X=(\Sigma, \mathcal{U})$ where $S=(\Sigma, \emptyset)$ is a surface with nodes without boundary and $\mathcal{U}$ is an analytic and maximal atlas of $\Sigma$. A stable Riemann surface is a Riemann surface with nodes whose parts have negative Euler characteristic. The reader can observe that $N(\Sigma)=N(\Sigma, 1)$. In this case, we define the genus of $\Sigma$ by

$$
g(\Sigma)=\frac{1}{2}(2+\# N(\Sigma)-\chi(\Sigma))
$$

A Klein surface with nodes is a triple $X=(\Sigma, \mathcal{D}, \mathcal{U})$ where $(\Sigma, \mathcal{D})$ is a surface with nodes and $\mathcal{U}$ is a dianalytic and maximal atlas of $\Sigma$.

Let $X$ be a Riemann or Klein surface with nodes where $N(\Sigma)=\left\{z_{i}\right\}_{i \in I}$. Let $f_{i}: U_{i} \rightarrow V_{i}$ be the charts, with

$$
V_{i}=V_{i, 1} \underset{w_{i}}{\sqcup} V_{i, 2}=\left(V_{i, 1} \times\{1\} \cup V_{i, 2} \times\{2\}\right) / \sim
$$

where $\sim$ is the identification $\left(w_{i}, 1\right) \sim\left(w_{i}, 2\right)$. We denote by

$$
U_{i}=U_{i, 1} \underset{z_{i}}{\sqcup} U_{i, 2} \quad \text { with } \quad U_{i, k}=f_{i}^{-1}\left(V_{i, k}\right)
$$

We take $\Sigma \backslash N(\Sigma)$ and construct $\hat{X}=(\hat{\Sigma}, \hat{\mathcal{U}})$ identifying $U_{i, k} \backslash\left\{z_{i}\right\}$ with $V_{i, k}$ using $f_{i}$ and we assign charts in the obvious way. We obtain a Riemann or Klein surface, $\hat{X}$, that, in general, is not connected and we call it the normalization of the Riemann or Klein surface with nodes $X$. The projection

$$
\begin{aligned}
p: \hat{\Sigma} & \rightarrow \Sigma \\
z & \mapsto z
\end{aligned}
$$

is an identification map and $\# p^{-1}(z)=2$ if and only if $z \in N(\Sigma, 1) \cup N(\Sigma, 3)$.

A map $f: \Sigma_{1} \rightarrow \Sigma_{2}$ between Riemann or Klein surfaces with nodes is a continuous map such that $f\left(\partial \Sigma_{1}\right) \subset \partial \Sigma_{2}$ and $f^{-1}\left(N\left(\Sigma_{2}, i\right)\right) \subset N\left(\Sigma_{1}, 1\right) \cup N\left(\Sigma_{1}, i\right)$. This map induces a unique continuous map $\hat{f}: \widehat{\Sigma_{1}} \rightarrow \widehat{\Sigma_{2}}$ satisfying $f \circ p_{1}=p_{2} \circ \hat{f}$ and is called the lifting of $f$.

A map $f: \Sigma_{1} \rightarrow \Sigma_{2}$ between Riemann or Klein surfaces with nodes is complete if $\hat{f}\left(p_{1}^{-1}(z)\right)=p_{2}^{-1}(f(z))$. Finally, a morphism between Riemann (resp. Klein) surfaces with nodes is an analytic (resp. dianalytic) and complete map $f: X_{1} \rightarrow X_{2}$ between Riemann (resp. Klein) surfaces with nodes. We have that $f$ is analytic (resp. antianalytic, dianalytic, ...) if and only if $\hat{f}$ is analytic (resp. antianalytic, dianalytic, ....). The homeomorphisms, isomorphisms and automorphisms are defined in the obvious way and we denote them by $\operatorname{Homeo}\left(X_{1}, X_{2}\right), \operatorname{Iso}\left(X_{1}, X_{2}\right)$ and $\operatorname{Aut}(X)$.

A symmetric Riemann surface with nodes is a pair $(X, \sigma)$ where $X$ is a Riemann surface with nodes and $\sigma: X \rightarrow X$ is an antianalytic involution. A map (resp. homeomorphism, morphism, antianalytic morphism, ...) $f:\left(X_{1}, \sigma_{1}\right) \rightarrow\left(X_{2}, \sigma_{2}\right)$ 
between symmetric Riemann surfaces with nodes is a map (resp. homeomorphism, morphism, antianalytic morphism, ...) $f: X_{1} \rightarrow X_{2}$ satisfying $f \circ \sigma_{1}=\sigma_{2} \circ f$.

We know that if $X$ is a Klein surface, then there exists a triple $\left(X_{c}, \pi_{c}, \sigma_{c}\right)$ such that $\left(X_{c}, \sigma_{c}\right)$ is a symmetric Riemann surface and $\pi_{c}: X_{c} \rightarrow X$ is an unbranched double cover satisfying $\pi_{c} \circ \sigma_{c}=\pi_{c}$. If $\left(X_{c}^{\prime}, \pi_{c}^{\prime}, \sigma_{c}^{\prime}\right)$ is another triple with the same property, then there exists a unique analytic isomorphism $f:\left(X_{c}^{\prime}, \sigma_{c}^{\prime}\right) \rightarrow\left(X_{c}, \sigma_{c}\right)$ between symmetric Riemann surfaces such that $\pi_{c}^{\prime}=\pi_{c} \circ f$. This triple, that is unique up to isomorphism, is called the complex double of $X$.

Let $Y$ be a Klein surface with nodes. We say that a triple $(X, \pi, \sigma)$ is a complex double of $Y$ if $(X, \sigma)$ is a symmetric Riemann surface with nodes and $\pi: X \rightarrow Y$ is an unbranched double cover satisfying $\pi \circ \sigma=\pi$ and $\pi(N(X))=N(Y)$. We say that two complex doubles, $\left(X_{1}, \pi_{1}, \sigma_{1}\right),\left(X_{2}, \pi_{2}, \sigma_{2}\right)$, of $Y$ are isomorphic if there is an isomorphism $f:\left(X_{1}, \sigma_{1}\right) \rightarrow\left(X_{2}, \sigma_{2}\right)$ between symmetric Riemann surfaces with nodes such that $\pi_{2} \circ f=\pi_{1}$.

If we have a Klein surface with nodes $X$, then there exist exactly $2^{\# N(X, 1)}$ triples $\left(X_{c}, \pi_{c}, \sigma_{c}\right)$ that are non-isomorphic complex doubles of $X$ (see [5]). We say that $X$ is a stable Klein surface if any one of its complex doubles is a stable symmetric Riemann surface. We define the algebraic genus of $X$ as the genus of any one of its complex doubles.

\section{Automorphism groups of Klein surfaces with nodes}

Let us recall some results about automorphism groups acting on Riemann and Klein surfaces. Let $X$ be a Riemann or Klein surface (with or without nodes) and let $G \leq \operatorname{Aut}(X)$. If $x \in X$, we denote the orbit of the point $x$ by $O_{x}=\{f(x) \mid f \in G\}$ and we construct the quotient space $X / G=\left\{O_{x} \mid x \in X\right\}$. We define the canonical projection

$$
\begin{aligned}
\pi: X & \rightarrow X / G \\
x & \mapsto O_{x} .
\end{aligned}
$$

Such projection defines an identification topology on $X / G: A \subset X / G$ is an open set if and only if $\pi^{-1}(A)$ is an open set. Hence, $\pi$ is an identification map. The topological space $X / G$ is the quotient of $X$ under the action of the group $G$.

The stabilizer of $x \in X$ is the subgroup

$$
G_{x}=\{f \in G \mid f(x)=x\} .
$$

If $U, V \subset X$, we denote by

$$
\begin{aligned}
G(U, V)= & \{f \in G \mid U \cap f(V) \neq \emptyset\}, \\
G_{U} & =G(U, U) .
\end{aligned}
$$

With this notation $G_{x}=G_{\{x\}}$.

We say that $G$ acts discontinuously on $X$ if for all $x \in X$ there exists a neighborhood $U \subset X$ of $x$ such that $G_{U}$ is finite. We have the following result: 
Proposition 2.1 ([1]). The following conditions are equivalent:

(i) $G$ acts discontinuously on $X$.

(ii) For all $x \in X, G_{x}$ is finite. Moreover, for any neighborhood $M \subset X$ of $x$, then there exists a connected neighborhood $U \subset M$ of $x$ satisfying $G_{U}=G_{x}$ and $f(U)=U$ if $f \in G_{x}$.

Let $X$ be a connected Klein surface (thus without nodes). We shall say that $G \leq \operatorname{Aut}(X)$ acts properly discontinuously on $X$ if the following conditions hold:

(i) $G$ acts discontinuously on $X$.

(ii) For all $x, y \in X$ such that $O_{x} \neq O_{y}$, there exist neighborhoods $U, V \subset X$ of $x$ and $y$ respectively, satisfying $G(U, V)=\emptyset$.

Let us recall a known theorem:

Theorem 2.2 ([1]). Let $X$ be a connected Klein surface and $G \leq \operatorname{Aut}(X)$ which acts properly discontinuously on $X$. Then, there exists a unique structure of Klein surface on $X / G$ such that $\pi: X \rightarrow X / G$ is a morphism.

This theorem is also satisfied if we consider non connected Klein surfaces. For this it suffices to apply it to the connected components of the Klein surface.

The situation changes when we try to apply this theorem to Klein surfaces with nodes. Let $\hat{X}=(\hat{\mathbb{C}} \times\{1\}) \cup(\hat{\mathbb{C}} \times\{2\})$ and $X=\hat{X} / \sim$ where $\sim$ is the identification $(0,1) \sim(0,2)$. Hence, $X$ is a Klein surface with nodes and $\hat{X}$ is its normalization. We define

$$
\begin{array}{cccc}
f: & X & \rightarrow & X \\
(z, 1) & \mapsto & (-z, 1), \\
(z, 2) & \mapsto & (\bar{z}, 2),
\end{array}
$$

which is an automorphism of $X$ satisfying $f^{2}=\operatorname{Id}_{X}$. If we take the subgroup $G=\langle f\rangle$ of $\operatorname{Aut}(X)$, then $X / G$ is homeomorphic to

$$
\left((\hat{\mathbb{C}} \times\{1\}) \cup\left(\widehat{\mathbb{C}^{+}} \times\{2\}\right)\right) / \sim_{G}
$$

where $\sim_{G}$ is the identification $(0,1) \sim_{G}(0,2)$. Although $G$ is a group that acts properly discontinuously on $X, X / G$ is not a surface with nodes because $[(0,1)]_{G}=[(0,2)]_{G}$ is not a node. This example motivates the following definition. Let $X$ be a Riemann or Klein surface with nodes and $f \in \operatorname{Aut}(X)$. We shall say that $f$ is dual on $x \in N(X, 1)$ if $f(x)=x$ and there exists a neighborhood $U=U_{1} \underset{x}{\sqcup_{2}} U_{2}$ of $x$ such that $f\left(U_{i}\right)=U_{i}$ and $\left.\hat{f}\right|_{\hat{U}}$ is a morphism that is neither analytic nor antianalytic, i.e., one of the maps $\left.\hat{f}\right|_{\widehat{U_{1}}}$ and $\left.\hat{f}\right|_{\widehat{U_{2}}}$ is analytic and the other is antianalytic. We shall say that $f$ is dual if there exists $x \in N(X, 1)$ such that $f$ is dual on $x$. We denote the set of dual automorphisms of $X$ by $\operatorname{Aut}^{d}(X)$.

Let $X$ be a Riemann or Klein surface with or without nodes. We shall say that $G \leq \operatorname{Aut}(X)$ acts properly discontinuously on $X$ if the following conditions hold: 
(i) $G$ acts discontinuously on $X$.

(ii) For all $x, y \in X$ such that $O_{x} \neq O_{y}$, there exist neighborhoods $U, V \subset X$ of $x$ and $y$ respectively, such that $G(U, V)=\emptyset$.

(iii) $G$ does not contain dual automorphisms.

Let $\hat{G}=\{\hat{f} \mid f \in G\}$ be the lifting of the group $G$, that is a subgroup of $\operatorname{Aut}(\hat{X})$. Let us look for the relation between these two groups.

Firstly, we suppose that $G$ acts properly discontinuously on $X$ and we denote by $p: \hat{X} \rightarrow X$ the projection.

Let $x \in \hat{X}$, then $y=p(x) \in X$ and there exists a neighborhood $U$ of $y$ such that $G_{U}$ is finite. We take the neighborhood $\hat{U}=p^{-1}(U)$ of $x$. If $\hat{f} \in \hat{G}_{\hat{U}}$, then

$$
\emptyset \neq p(\hat{U} \cap \hat{f}(\hat{U})) \subset p(\hat{U}) \cap p \circ \hat{f}(\hat{U})=p(\hat{U}) \cap f(p(\hat{U}))=U \cap f(U)
$$

and $f \in G_{U}$. As $G_{U}$ is finite, then $\hat{G}_{\hat{U}}$ is also finite and $\hat{G}$ acts discontinuously on $X$.

Let $x, y \in \hat{X}$ such that $O_{x} \neq O_{y}$ and we take $z=p(x)$ and $w=p(y)$. There are two possibilities:

(i) If $z=w$, then we take a connected neighborhood $U$ of $z$ such that $G_{U}=$ $G_{z}$. Thus, $p^{-1}(U)=\widehat{U_{x}} \sqcup \widehat{U_{y}}$ where $\widehat{U_{x}}, \widehat{U_{y}}$ are connected neighborhoods of $x$ and $y$ respectively. Let $\hat{f} \in \hat{G}$ such that $\widehat{U_{x}} \cap \hat{f}\left(\widehat{U_{y}}\right) \neq \emptyset$, then $\hat{f}\left(\widehat{U_{y}}\right)=\widehat{U_{x}}$, $f(U)=U, f(z)=z$, and $\hat{f}(y)=x$. That is impossible because $O_{x} \neq O_{y}$. Hence, $\hat{G}\left(\widehat{U_{x}}, \widehat{U_{y}}\right)=\emptyset$.

(ii) If $z \neq w$, then we take open neighborhoods $U_{z}, U_{w}$ of $z$ and $w$ respectively such that $G\left(U_{z}, U_{w}\right)=\emptyset$. If we call $\widehat{U_{x}}=p^{-1}\left(U_{z}\right)$ and $\widehat{U_{y}}=p^{-1}\left(U_{w}\right)$, then, for all $\hat{f} \in \hat{G}$

$$
p\left(\widehat{U_{x}} \cap \hat{f}\left(\widehat{U_{y}}\right)\right) \subset p\left(\widehat{U_{x}}\right) \cap p \circ \hat{f}\left(\widehat{U_{y}}\right)=p\left(\widehat{U_{x}}\right) \cap f \circ p\left(\widehat{U_{y}}\right)=U_{z} \cap f\left(U_{w}\right)=\emptyset
$$

and again $\hat{G}\left(\widehat{U_{x}}, \widehat{U_{y}}\right)=\emptyset$. Hence, $\hat{G}$ acts properly discontinuously on $X$.

If $\hat{G}$ acts properly discontinuously on $\hat{X}$ and $G$ does not contain dual automorphisms, then we can prove, in a similar way, that $G$ acts properly discontinuously on $X$. Then we have the following proposition:

Proposition 2.3. Let $X$ be a Riemann or Klein surface with nodes and $G \leq \operatorname{Aut}(X)$ a group that does not contain dual automorphisms. The group $G$ acts properly discontinuously on $X$ if and only if $\hat{G}$ acts properly discontinuously on $\hat{X}$. 


\section{Quotients of Klein surfaces with nodes}

Let $X$ be a Klein surface with nodes and let $G \leq \operatorname{Aut}(X)$ be a group acting properly discontinuously on $X$. Since $\hat{G}$ acts properly discontinuously on $\hat{X}$, then there exists a unique structure of Klein surface on $\hat{X} / \hat{G}$ such that

$$
\begin{aligned}
\hat{\pi}: \hat{X} & \rightarrow \hat{X} / \hat{G} \\
x & \mapsto \hat{O}_{x}
\end{aligned}
$$

is a morphism. We have the following projections:

$$
\begin{aligned}
& p: \hat{X} \rightarrow X \quad \pi: X \rightarrow X / G \\
& x \mapsto p(x) \quad x \mapsto O_{x} .
\end{aligned}
$$

and we define $p_{G}: \hat{X} / \hat{G} \rightarrow X / G$ by $p_{G}\left(\hat{O}_{x}\right)=O_{p(x)}$. It is easy to check that $p_{G}$ is a well defined map and the following diagram is commutative:

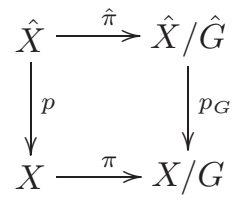

Let us see that $p_{G}$ is an identification map. If $B \subset X / G$ is an open set, then $\hat{\pi}^{-1} \circ p_{G}^{-1}(B)=(\pi \circ p)^{-1}(B)$ is also an open set and, since $\hat{\pi}$ is an identification map, $p_{G}^{-1}(B)$ is an open set and hence $p_{G}$ is continuous. On the other hand, since $p$ and $\pi$ are identification maps, so is $p_{G}$.

Before proving the theorem establishing that $X / G$ is a Klein surface with nodes, let us prove a lemma.

Lemma 3.1. Let $\Sigma$ be a topological space, let $\hat{X}=(\hat{\Sigma}, \hat{\mathcal{U}})$ be a Klein surface and let $p: \hat{X} \rightarrow \Sigma$ be an identification map satisfying:

(i) For each $x \in \Sigma, \# p^{-1}(x) \in\{1,2\}$.

(ii) The set $A^{\prime}$ of cluster points of the set $A=\left\{x \in \Sigma \mid \# p^{-1}(x)=2\right\}$ is empty.

(iii) For each $x \in A$ either $p^{-1}(x) \subset \partial \hat{X}$ or $p^{-1}(x) \cap \partial \hat{X}=\emptyset$.

Then, for each subset $B \subset \Sigma \backslash p(\partial \hat{X})$ disjoint with $A$ and such that $B^{\prime}=\emptyset$, there exists a unique structure of Klein surface with nodes, $X$, on $\Sigma$ such that $N(X, 2)=B$, $\hat{X}$ is its normalization and $p: \hat{X} \rightarrow X$ is the projection.

Proof. Let $x$ be in $\Sigma$. We distinguish two cases:

(a) If $x \notin A$, then $p^{-1}(x)=\{y\}$ and there exists an open neighborhood $\hat{V} \subset \hat{\Sigma}$ of $y$ such that $\hat{V} \cap p^{-1}(A)=\emptyset$. We take a chart $(\hat{V}, \varphi) \in \hat{\mathcal{U}}$ and then $\left(p(\hat{V}), \varphi \circ p^{-1}\right)$ is a chart on $x$. 
(b) If $x \in A$ then either $p^{-1}(x) \cap \partial \hat{X}=\emptyset$ or $p^{-1}(x) \subset \partial \hat{X}$. Hence $p^{-1}(x)=\left\{y_{1}, y_{2}\right\}$ and there exist disjoint open neighborhoods $\widehat{V_{1}}, \widehat{V_{2}} \subset \hat{\Sigma}$ of $y_{1}, y_{2}$ respectively satisfying $\widehat{V}_{i} \cap p^{-1}(A)=\left\{y_{i}\right\}$. We take the charts $\left(\widehat{V}_{i}, \varphi_{i}\right) \in \hat{\mathcal{U}}$ with $\varphi_{i}: \widehat{V}_{i} \rightarrow U_{i}$ and $\varphi_{i}\left(y_{i}\right)=0$. Now we define $\varphi: \widehat{V_{1}} \cup \widehat{V_{2}} \rightarrow U_{1} \underset{0}{\cup} U_{2}$ by $\varphi(z)=\varphi_{i}(z)$ if $z \in \widehat{V}_{i}$. Hence $\left(p\left(\widehat{V_{1}} \cup \widehat{V_{2}}\right), \varphi \circ p^{-1}\right)$ is a chart on $x$.

This way we obtain a dianalytic atlas $\tilde{\mathcal{U}}$ that induces a dianalytic and maximal atlas $\mathcal{U}$ on $\Sigma$. Thus $X=(\Sigma, B, \mathcal{U})$ is a Klein surface with nodes, $\hat{X}$ is its normalization and $p: \hat{X} \rightarrow X$ is the projection.

If there are two structures, $X_{1}, X_{2}$, of Klein surface with nodes on $\Sigma$ with the conditions of the lemma, then $\operatorname{Id}_{\Sigma}: X_{1} \rightarrow X_{2}$ is a homeomorphism between Klein surfaces with nodes. Since its lifting $\widehat{\operatorname{Id}_{\Sigma}}=\operatorname{Id}_{\hat{X}}: \hat{X} \rightarrow \hat{X}$ is an isomorphism, then $f$ is also an isomorphism and this proves the uniqueness.

Let us verify that $p_{G}: \hat{X} / \hat{G} \rightarrow X / G$ satisfies the three hypotheses of the lemma.

Let $z \in X / G$ and we take $x \in \pi^{-1}(z)$. If $p^{-1}(x)=\{y\}$, then

$$
p_{G} \circ \hat{\pi}(y)=\pi \circ p(y)=\pi(x)=z
$$

and $\{\hat{\pi}(y)\} \subset p_{G}^{-1}(z)$.

Let $a \in p_{G}^{-1}(z)$, then there exists $b \in \hat{X}$ such that $\hat{\pi}(b)=a$. But

$$
\pi(x)=z=p_{G}(a)=p_{G} \circ \hat{\pi}(b)=\pi \circ p(b) .
$$

Thus, there exists $g \in G$ satisfying $x=g \circ p(b)=p \circ \hat{g}(b)$ and therefore $\hat{g}(b)=y$ and

$$
\hat{\pi}(y)=\hat{\pi} \circ \hat{g}(b)=\hat{\pi}(b)=a .
$$

Hence $a \in\{\hat{\pi}(y)\}$ and $p_{G}^{-1}(z)=\{\hat{\pi}(y)\}$.

On the other hand, if $p^{-1}(x)=\left\{y_{1}, y_{2}\right\}$ then $p_{G}^{-1}(z)=\left\{\hat{\pi}\left(y_{1}\right), \hat{\pi}\left(y_{2}\right)\right\}$ and the first condition is satisfied.

If $z \in A=\left\{z \in X / G \mid \# p_{G}^{-1}(z)=2\right\}$, then $z \in \pi(N(X))$. Hence it is sufficient to prove that the set of cluster points of $\pi(N(X))$ is empty. Let $z \in X / G$ and $x \in \pi^{-1}(z)$ and we take $U$, that is an open neighborhood of $x$ satisfying $(U \backslash\{x\}) \cap N(X)=\emptyset$. Then, $\pi(U)$ is an open set because $\pi^{-1}(\pi(U))=\bigcup_{g \in G} g(U)$. Hence $\pi(U)$ is an open neighborhood of $z$ such that $(\pi(U)-\{z\}) \cap \pi(N(X))$ is empty.

To prove the third condition, let $z \in A, x \in \pi^{-1}(z)$ and $p^{-1}(x)=\left\{y_{1}, y_{2}\right\}$. Then $p_{G}^{-1}(z)=\left\{\hat{\pi}\left(y_{1}\right), \hat{\pi}\left(y_{2}\right)\right\}$ and we suppose that $\hat{\pi}\left(y_{1}\right) \in \partial(\hat{X} / \hat{G})$ and $\hat{\pi}\left(y_{2}\right) \notin \partial(\hat{X} / \hat{G})$. In this situation $y_{2} \notin \partial \hat{X}$ and $x \notin \partial X$. Hence $x$ is a conic node of $X$ and so $y_{1} \notin \partial \hat{X}$. Since $\hat{\pi}\left(y_{1}\right) \in \partial(\hat{X} / \hat{G})$ and $\hat{\pi}\left(y_{2}\right) \notin \partial(\hat{X} / \hat{G})$, then there exists $g \in G$ dual on $x$, which is a contradiction. Therefore, the three hypotheses of the lemma are satisfied.

The following step for giving $X / G$ a structure of Klein surface with nodes is to decide what could be the inessential nodes of this new surface. First of all, let us 
recall how these nodes appear (see [5]). A Klein surface is the quotient space of a Riemann surface by an antianalytic involution. Let $X$ be a Riemann surface with nodes, $\sigma: X \rightarrow X$ be an antianalytic involution, $z \in N(X)$ and $V_{1}, V_{2} \subset X$ be two disks such that $U=V_{1} \underset{z}{\sqcup} V_{2}$ is a neighborhood of $z$. If $\sigma\left(V_{1}\right)=V_{2}$, then $U /\langle\sigma\rangle \simeq V_{1}$ and $O_{z}$ is an inessential node of $X /\langle\sigma\rangle$.

Let $X$ be a Klein surface with nodes, $g \in \operatorname{Aut}(X)$ and $p: \hat{X} \rightarrow X$ be the projection. We shall say that $x \in N(X, 1)$ is an inessential node of $X$ with respect to $g$ if $p^{-1}(x)=\left\{x_{1}, x_{2}\right\}$ and $\hat{g}\left(x_{1}\right)=x_{2}$. In this situation $\hat{g}\left(x_{2}\right)=x_{1}$. If $G \leq \operatorname{Aut}(X)$, we denote the set of inessential nodes of $X$ with respect to some $g$ of $G$ by $N_{G}(X, 2)$. The union of the set of inessential nodes of $X$ with the image of $N_{G}(X, 2)$ under the projection $\pi: X \rightarrow X / G$ is the admissible set of inessential nodes of the new surface $X / G$.

Let $D \subset N_{G}(X, 2) \cup N(X, 2)$ and we take

$$
B=\pi(D) \backslash \partial(X / G)=\pi(D) \backslash p_{G}(\partial(\hat{X} / \hat{G})) .
$$

Since $D \subset N(X)$ and the set of cluster points of $\pi(N(X))$ is empty so is the set of cluster points of $\pi(D)$. Finally, if $x \in D$ it is easy to prove that $\# p_{G}^{-1}(\pi(x))=1$. Then, applying Lemma 3.1, there exists a unique structure of Klein surface on $X / G$ satisfying $N(X / G, 2)=\pi(D) \backslash \partial(X / G), \hat{X} / \hat{G}$ is the normalization of $X / G$ and $p_{G}: \hat{X} / \hat{G} \rightarrow X / G$ is the projection.

Now we can prove the following theorem:

Theorem 3.2. Let $X$ be a Klein surface with nodes and $G \leq \operatorname{Aut}(X)$ a group that acts properly discontinuously on $X$. Then, for each $D \subset N_{G}(X, 2) \cup N(X, 2)$ there exists a unique structure of Klein surface with nodes on $X / G$ such that $\pi: X \rightarrow X / G$ is a morphism and $N(X / G, 2)=\pi(D)-\partial(X / G)$.

Proof. Using the previous constructions, we only have to prove that $\pi$ is a morphism and the uniqueness. The projection $\pi: X \rightarrow X / G$ satisfies the following properties:

(i) $\pi(\partial X) \subset \partial(X / G)$.

Let $x \in \partial X$ and $z \in p^{-1}(x)$, then $z \in \partial \hat{X}$ and $\hat{\pi}(z) \in \partial(\hat{X} / \hat{G})$. Hence, $p_{G} \circ \hat{\pi}(z) \in \partial(X / G)$, but

$$
\pi(x)=\pi \circ p(z)=p_{G} \circ \hat{\pi}(z) \in \partial(X / G)
$$

and then $\pi(\partial X) \subset \partial(X / G)$.

(ii) $\pi^{-1}(N(X / G, 2)) \subset N(X, 1) \cup N(X, 2)$.

Let $x \in \pi^{-1}(N(X / G, 2))$, then there exists $y \in D \subset N(X, 1) \cup N(X, 2)$ satisfying $\pi(x)=\pi(y)$. Thus, there exists $g \in G$ such that $x=g(y) \in N(X, 1) \cup N(X, 2)$. Hence $\pi^{-1}(N(X / G, 2)) \subset N(X, 1) \cup N(X, 2)$. 
(iii) $\pi^{-1}(N(X / G, 3)) \subset N(X, 1) \cup N(X, 3)$.

Let $x \in \pi^{-1}(N(X / G, 1) \cup N(X / G, 3))$. Let us suppose that $\# p^{-1}(x)=1$, then $\# p_{G}^{-1}(\pi(x))=1$. This is impossible because $\pi(x) \in N(X)$. Thus, $\# p^{-1}(x)=2$ and $x \in N(X, 1) \cup N(X, 3)$. Hence $\pi^{-1}(N(X / G, 3)) \subset N(X, 1) \cup N(X, 3)$.

(iv) $\pi^{-1}(N(X / G, 1)) \subset N(X, 1)$.

Let $x \in \pi^{-1}(N(X / G, 1))$. As in the previous point, $x \in N(X, 1) \cup N(X, 3)$. If $x \in N(X, 3)$, then $x \in \partial X$ and $\pi(x) \in \partial(X / G)$. This is impossible because $\partial(X / G) \cap N(X / G, 1)=\emptyset$. Hence $x \in N(X, 1)$ and we conclude that $\pi^{-1}(N(X / G, 1)) \subset N(X, 1)$.

(v) $\pi$ is complete.

Let $x \in N(X, 1) \cup N(X, 3)$ such that $z=\pi(x) \in N(X / G, 1) \cup N(X / G, 3)$. Thus $p^{-1}(x)=\left\{y_{1}, y_{2}\right\}$ and $p_{G}^{-1}(z)=\left\{\hat{\pi}\left(y_{1}\right), \hat{\pi}\left(y_{2}\right)\right\}$. But, since $z$ is a conic or a boundary node of $X / G$, then $\#\left\{\hat{\pi}\left(y_{1}\right), \hat{\pi}\left(y_{2}\right)\right\}=2$ and $\# \hat{\pi}\left(p^{-1}(x)\right)=2$. Hence $\pi$ is complete.

Thus, $\pi: X \rightarrow X / G$ is a map between Klein surfaces with nodes. Let us recall the following commutative diagram

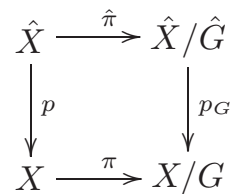

Since $\hat{\pi}$ is the lifting of $\pi$ and $\hat{\pi}$ is a morphism, so is $\pi$.

To prove the uniqueness, let us suppose that $X / G$ admits two structures of Klein surfaces with nodes, $(X / G)_{1},(X / G)_{2}$, such that the projections $\pi_{i}: X \rightarrow(X / G)_{i}$ are morphisms and $N\left((X / G)_{i}, 2\right)=\pi_{i}(D)-\partial(X / G)_{i}$.

We take the normalization $(\hat{X} / \hat{G})_{i}$ of $(X / G)_{i}$, the projection $p_{i}:(\hat{X} / \hat{G})_{i} \rightarrow(X / G)_{i}$ and the lifting $\widehat{\pi}_{i}: \hat{X} \rightarrow(\hat{X} / \hat{G})_{i}$ of $\pi_{i}$. As $\pi_{i}$ is a morphism, then $\widehat{\pi}_{i}$ is also a morphism. Hence $\operatorname{Id}_{\hat{X} / \hat{G}}:(\hat{X} / \hat{G})_{1} \rightarrow(\hat{X} / \hat{G})_{2}$ is an isomorphism between Klein surfaces. We have that $\operatorname{Id}_{X / G}:(X / G)_{1} \rightarrow(X / G)_{2}$ is a homeomorphism and hence:

- $\operatorname{Id}_{X / G}\left(\partial(X / G)_{1}\right)=\partial(X / G)_{2}$,

- $\operatorname{Id}_{X / G}\left(N\left((X / G)_{1}, i\right)\right)=N\left((X / G)_{2}, i\right), \quad i=1,3$.

Since $N\left((X / G)_{i}, 2\right)=\pi_{i}(D)-\partial(X / G)_{i}$, then

$$
\operatorname{Id}_{X / G}\left(N\left((X / G)_{1}, 2\right)\right)=N\left((X / G)_{2}, 2\right),
$$

and $\operatorname{Id}_{X / G}$ is a map between Klein surfaces with nodes and $\operatorname{Id}_{\hat{X} / \hat{G}}$ is its lifting. Since $\operatorname{Id}_{\hat{X} / \hat{G}}$ is an isomorphism, so is $\operatorname{Id}_{X / G}$. Therefore $(X / G)_{1},(X / G)_{2}$ are the same structure of Klein surface with nodes over $X / G$. 
Corollary 3.3. Let $X$ be a Klein surface with nodes and $G \leq \operatorname{Aut}(X)$ a group that acts properly discontinuously on $X$. Thus, there exists a unique structure of Klein surface with nodes on $X / G$ such that $\pi: X \rightarrow X / G$ is a morphism and $N(X / G, 2)=$ $\pi\left(N_{G}(X, 2) \cup N(X, 2)\right)-\partial(X / G)$.

From here on, by the Klein surface with nodes $X / G$ we mean the surface of the previous corollary.

Let us deal now with the quotients of Riemann surfaces with nodes. In this case we can distinguish between analytic and antianalytic automorphisms, and we denote by

$\operatorname{Aut}(X)=\{$ analytic automorphisms of the Riemann surface with nodes $X\}$,

$\operatorname{Aut}^{-}(X)=\{$ antianalytic automorphisms of the Riemann surface with nodes $X\}$, $\operatorname{Aut}^{ \pm}(X)=\{$ analytic and antianalytic automorphisms of the Riemann surface with nodes $X\}$.

If $G$ is a subgroup of $\operatorname{Aut}^{ \pm}(X)$ and we denote by $G^{-}=G \cap \operatorname{Aut}^{-}(X)$, then $N_{G^{-}}(X, 2) \subset N_{G}(X, 2)$ and we have:

Theorem 3.4. Let $X$ be a Riemann surface with nodes and $G \leq \operatorname{Aut}^{ \pm}(X)$ a group that acts properly discontinuously on $X$. Then, for each $D \subset N_{G^{-}}(X, 2)$ there exists a unique structure of Klein surface with nodes on $X / G$ such that $\pi: X \rightarrow X / G$ is a morphism and $N(X / G, 2)=\pi(D)-\partial(X / G)$.

By the Klein surface with nodes $X / G$ we mean the Klein surface with nodes such that $N(X / G, 2)=\pi\left(N_{G^{-}}(X, 2)\right)-\partial(X / G)$.

Finally, if $X$ is a Riemann surface with nodes and $G \leq \operatorname{Aut}(X)$, then $N_{G^{-}}(X, 2)$ is empty and we have the following theorem:

Theorem 3.5. Let $X$ be a Riemann surface with nodes and $G \leq \operatorname{Aut}(X)$ a group that acts properly discontinuously on $X$. Then, there exists a unique structure of Riemann surface with nodes on $X / G$ such that $\pi: X \rightarrow X / G$ is a morphism.

It is easy to see that if $X$ is a Riemann or Klein surface with nodes and $G$ is a finite subgroup of $\operatorname{Aut}(X)$ without dual automorphisms, then $G$ acts properly discontinuously on $X$. Moreover, if $X$ is a compact and connected stable Riemann or Klein surface of genus $g(X) \geq 2$, then $\operatorname{Aut}(X)$ is finite (see [4]). Combining these ideas we have the following corollaries:

Corollary 3.6. Let $X$ be a compact and connected stable Riemann surface of genus $g(X) \geq 2$ and let $G$ be a subgroup of $\operatorname{Aut}^{ \pm}(X)$ (resp. Aut $(X)$ ). Then, there exists a structure of Klein (resp. Riemann) surface with nodes on $X / G$ such that the projection $\pi: X \rightarrow X / G$ is a morphism. 
Corollary 3.7. Let $X$ be a compact and connected stable Klein surface of genus $g(X) \geq 2$ and let $G$ be a subgroup of $\operatorname{Aut}(X)$ without dual automorphisms. Then, there exists a structure of Klein surface with nodes on $X / G$ such that the projection $\pi: X \rightarrow X / G$ is a morphism.

\section{Riemann-Hurwitz's Formula}

Let $f: X \rightarrow Y$ be a morphism between compact and connected Riemann surfaces with nodes which is non-constant on the parts of $X$ and let $x$ be in $X$. If $p_{X}: \hat{X} \rightarrow X$ and $p_{Y}: \hat{Y} \rightarrow Y$ are the projections we define the multiplicity of $f$ at $x$ by

$$
\operatorname{mult}_{x}(f)= \begin{cases}\operatorname{mult}_{z}(\hat{f}) & \text { if } p_{X}^{-1}(x)=\{z\}, \\ \operatorname{mult}_{z_{1}}(\hat{f})+\operatorname{mult}_{z_{2}}(\hat{f}) & \text { if } p_{X}^{-1}(x)=\left\{z_{1}, z_{2}\right\} .\end{cases}
$$

If $y \in Y \backslash N(Y)$, we define the degree of $f$ at $y$ by

$$
d_{y}(f)=\sum_{x \in f^{-1}(y)} \operatorname{mult}_{x}(f)=d_{p_{Y}^{-1}(y)}(\hat{f})
$$

which is a function that is constant on the parts of $Y$. If $\hat{Y}=\bigcup_{i=1}^{n} \widehat{Y}_{i}$ where $\widehat{Y}_{i}$ are the connected components of $\hat{Y}$ and $w_{i} \in \widehat{Y}_{i} \backslash p_{Y}^{-1}(N(Y))$, then we define the degree of $f$ as $\operatorname{deg}(f)=\left(d_{p_{Y}\left(w_{1}\right)}(f), \ldots, d_{p_{Y}\left(w_{n}\right)}(f)\right)$. If $d_{p_{Y}\left(w_{1}\right)}(f)=\cdots=d_{p_{Y}\left(w_{n}\right)}(f)$, then we define the degree of $f$ as $\operatorname{deg}(f)=d_{p_{Y}\left(w_{1}\right)}(f)$.

Let $\widehat{X}_{i}=\hat{f}^{-1}\left(\widehat{Y}_{i}\right)$. If we apply Riemann-Hurwitz's Formula to the morphism $\widehat{f}_{i}=\left.\hat{f}\right|_{\widehat{X}_{i}}: \widehat{X}_{i} \rightarrow \widehat{Y}_{i}$, we obtain

$$
-\chi\left(\widehat{X}_{i}\right)=-\operatorname{deg}\left(\widehat{f}_{i}\right) \chi\left(\widehat{Y}_{i}\right)+\sum_{z \in \widehat{X}_{i}}\left[\operatorname{mult}_{z}\left(\widehat{f}_{i}\right)-1\right],
$$

where $\chi$ denotes the Euler characteristic.

Since $\chi(\hat{X})=\sum_{i=1}^{n} \chi\left(\widehat{X_{i}}\right)=2 \# N(X)+2-2 g(X)$ and using the definition of the multiplicity of $f$ at $x$ we conclude that

$$
\begin{aligned}
2 g(X)-2-2 \# N(X)=-\sum_{i=1}^{n} & \operatorname{deg}\left(\widehat{f}_{i}\right) \chi\left(\widehat{Y}_{i}\right) \\
& +\sum_{x \notin N(X)}\left[\operatorname{mult}_{x}(f)-1\right]+\sum_{x \in N(X)}\left[\operatorname{mult}_{x}(f)-2\right] .
\end{aligned}
$$

We have proved the following theorem:

Theorem 4.1 (Riemann-Hurwitz's Formula). Let $f: X \rightarrow Y$ be a morphism between compact and connected Riemann surfaces with nodes, which is non-constant 
on the parts of $X$ and such that $\operatorname{deg}(f) \in \mathbb{N}$. Then

$$
\begin{aligned}
2 g(X)-2-2 \# N(X)=\operatorname{deg}(f) & 2 g(Y)-2-2 \# N(Y)) \\
& +\sum_{x \notin N(X)}\left[\operatorname{mult}_{x}(f)-1\right]+\sum_{x \in N(X)}\left[\operatorname{mult}_{x}(f)-2\right] .
\end{aligned}
$$

Let $G$ be a group of automorphisms acting properly discontinuously on the compact and connected Riemann surface with nodes $X$. Suppose that $y \in Y=X / G$ is a branch point of the quotient map $\pi: X \rightarrow X / G$. Let $x_{1}, \ldots, x_{s}$ be the points of $X$ lying above $y$. Then all of them are in the same orbit and hence $\pi$ have the same multiplicity at all of them, say $r$. Using previous notation, we denote by

$$
\widehat{G_{i}}=\left\{\left.\hat{g}\right|_{\widehat{X}_{i}} \in \operatorname{Aut}\left(\widehat{X_{i}}\right) \mid g \in G\right\} .
$$

We have three possibilities:

(i) If $x_{i} \notin N(X)$, and $y \in p_{Y}\left(\widehat{Y}_{i}\right)$, then $s=\left|\widehat{G_{i}}\right| / r$ and

$$
\sum_{i=1}^{s}\left[\operatorname{mult}_{x_{i}}(f)-1\right]=\left|\widehat{G_{i}}\right|-\frac{\left|\widehat{G_{i}}\right|}{r} .
$$

(ii) If $x_{i} \in N(X)$ and $y \in p_{Y}\left(\widehat{Y}_{i}\right) \backslash N(Y)$, then $r$ is even, $s=\left|\widehat{G_{i}}\right| / r$ and

$$
\sum_{i=1}^{s}\left[\operatorname{mult}_{x_{i}}(f)-2\right]=\left|\widehat{G_{i}}\right|-2 \frac{\left|\widehat{G_{i}}\right|}{r} .
$$

(iii) If $x_{i} \in N(X)$ and $y \in p_{Y}\left(\widehat{Y_{i}}\right) \cap p_{Y}\left(\widehat{Y_{j}}\right)$, then $r=r_{i}+r_{j}, s=\left|\widehat{G_{i}}\right| / r_{i}=\left|\widehat{G_{j}}\right| / r_{j}$ and

$$
\sum_{i=1}^{s}\left[\operatorname{mult}_{x_{i}}(f)-2\right]=\left|\widehat{G_{i}}\right|-\frac{\left|\widehat{G_{i}}\right|}{r_{i}}+\left|\widehat{G_{j}}\right|-\frac{\left|\widehat{G_{j}}\right|}{r_{j}} .
$$

We can summarize these observations in the following corollary:

Corollary 4.2. Let $G$ be a group acting properly discontinuously on the compact and connected Riemann surface with nodes $X$, with quotient map $\pi: X \rightarrow Y=X / G$. Suppose that there are $k_{i}$ branch points $y_{i_{1}}, \ldots, y_{i_{k_{i}}}$ in $p_{Y}\left(\widehat{Y}_{i}\right)$, with $\pi$ having multiplicity $r_{i_{j}}$ at the points above $y_{i_{j}}$. Then

$$
2 g(X)-2-2 \# N(X)=\sum_{i=1}^{n}\left[\left|\widehat{G}_{i}\right|\left[-\chi\left(\widehat{Y}_{i}\right)+\sum_{j=1}^{k_{i}}\left(1-\frac{\varepsilon_{i_{j}}}{r_{i_{j}}}\right)\right]\right]
$$

with

$$
\varepsilon_{i_{j}}= \begin{cases}2 & \text { if } y_{i_{j}} \notin N(Y) \text { and } \pi^{-1}\left(y_{i_{j}}\right) \subset N(X), \\ 1 & \text { otherwise. }\end{cases}
$$


In a forthcoming paper we will use this last corollary to solve both the so called maximum order and minimum genus problems for Riemann surfaces with nodes. The first is the computation, for each fixed integer $g$ greater than 1 , of the maximum integer $N=N(g)$ such that there exists a compact Riemann surface with nodes, of genus $g$, which admits an automorphism of order $N$. The second is the computation, for each fixed integer $N$ greater than 1, of the minimum integer $g=g(N)$ such that there exists a compact Riemann surface with nodes, of genus $g$, which admits an automorphism of order $N$.

\section{References}

[1] E. Bujalance, J. J. Etayo, J. M. Gamboa, and G. Gromadzki, Automorphism groups of compact bordered Klein surfaces, Lecture Notes in Mathematics, vol. 1439, Springer-Verlag, Berlin, 1990.

[2] P. Deligne and D. Mumford, The irreducibility of the space of curves of given genus, Inst. Hautes Études Sci. Publ. Math. (1969), no. 36, 75-109.

[3] I. Garijo, Complex doubles of Klein surfaces with nodes and the compactification of the moduli spaces of real algebraic curves, UNED, May 2005, preprint.

[4] Superficies de Riemann y Klein con nodos, Ph. D. Thesis, UNED, 2000.

[5] The double covers of Klein surfaces with nodes, May 2005, ArXiv:math.CV/0505182v1, preprint.

[6] M. Kontsevich, Intersection theory on the moduli space of curves and the matrix Airy function, Comm. Math. Phys. 147 (1992), no. 1, 1-23.

[7] S. K. Lando and A. K. Zvonkin, Graphs on surfaces and their applications, Encyclopaedia of Mathematical Sciences, vol. 141, Springer-Verlag, Berlin, 2004.

[8] R. Miranda, Algebraic curves and Riemann surfaces, Graduate Studies in Mathematics, vol. 5, American Mathematical Society, Providence, RI, 1995.

[9] M. Seppälä, Moduli spaces of stable real algebraic curves, Ann. Sci. École Norm. Sup. (4) 24 (1991), no. 5, 519-544.

[10] D. Zvonkine, Strebel differentials on stable curves and Kontsevich's proof of Witten's conjecture, Jan. 2004, arXiv:math.AG/0209071v2, preprint. 